\title{
Receptor Tyrosine-Protein Kinase erbB-2
}

National Cancer Institute

\section{Source}

National Cancer Institute. Receptor Tyrosine-Protein Kinase erbB-2. NCI Thesaurus.

Code C17319.

Receptor tyrosine-protein kinase erbB-2 (1255 aa, 138 kDa) is encoded by the human ERBB2 gene. This protein is involved in cell proliferation, tyrosine phosphorylation and signal transduction. 